\title{
A FORMULA BY V. M. ADAMJAN, D. Z. AROV AND M. G. KREIN
}

\section{ARNE STRAY}

\begin{abstract}
For $F$ in $L^{\infty}$ of the unit circle, Adamjan, Arov and Krein obtained a formula describing all functions in the coset $F+H^{\infty}$ of norms not exceeding 1. A proof of this result using classical ideas of Nevanlinna and Schur is given.
\end{abstract}

Let $F$ be a bounded measurable function on the unit circle $T=\{z:|z|=1\}$; and form the set

$$
B_{F}=\left\{F-h: h \in H^{\infty},\|F-h\|_{\infty}<1\right\},
$$

where $H^{\infty}$ denotes the space of bounded measurable functions on $T$ which extend to be analytic in $D=\{z:|z|<1\}$ by means of the Poisson integral formula. The norm " \|\|$_{\infty}$ " denotes the usual essential supremum on $T$.

In a series of papers [1], [2], [3], V. M. Adamjan, D. Z. Arov and M. G. Krein have obtained very detailed information about the set $B_{F}$. Their proofs use the theory of bounded operators on Hilbert space and basic knowledge about the classical Hardy spaces.

The object of this paper is to give an elementary proof of one of their results:

Theorem (Adamuan, Arov and Krein). Suppose $B_{F}$ contains at least two functions. Then there are analytic functions $p$ and $q$ in $D$ such that

$$
B_{F}=\left\{\frac{\bar{q}-\bar{p} w}{p-q w}: w \in H^{\infty},\|w\|_{\infty}<1\right\} .
$$

REMARK. The functions $p$ and $q$ have boundary values defined almost everywhere on $T$, and in the above description of $B_{F}$, all functions should be considered on the unit circle $T$ only. Two special cases of the above problem should be mentioned:

(i) I. Schur's coefficient problem [9], [10],

(ii) R. Nevanlinna's work in [7], [8] on interpolation by bounded analytic functions.

What we do here is to prove the above theorem by combining ideas of Schur and Nevanlinna. In this way we obtain a proof completely based on classical complex analysis.

This work was done while the author was visiting the University of Maryland, College Park, as a research associate. I would like to express my gratitude to the

Received by the editors October 28, 1980.

1980 Mathematics Subject Classification. Primary 30D50; Secondary 30E05. 
Mathematics Department for their hospitality. In particular I would like to thank professors Alice Chang and Maurice Heins for stimulating contact concerning the problems studied here.

Proof of THE THEOREM. We let $\sigma_{N}$ denote the $N$ th Fejer kernel and form the trigonometric polynomials $F_{N}=F * \sigma_{N}, N=1,2, \ldots$ As $B_{F}$ corresponds to $F$, we define $B_{N}=\left\{F_{N}-h: h \in H^{\infty},\left\|F_{N}-h\right\|_{\infty}<1\right\}$. If $N$ is sufficiently large, $B_{N}$ has at least two members. The following formula is due to I. Schur [9], [10] although it is not explicitly stated there:

$$
B_{N}=\frac{\bar{q}_{N}-\bar{p}_{N} w}{p_{N}-q_{N} w}, \quad w \in H^{\infty},\|w\|_{\infty}<1,
$$

where $p_{N}$ and $q_{N}$ are polynomials constructed by the Schur algorithm [10] in terms of the Fourier coefficients of $F_{N}$.

We list some properties of $p_{N}$ and $q_{N}$ which are part of Schur's construction:

$$
\begin{aligned}
& p_{N}(z) \neq 0, \quad|z|<1, \\
& q_{N}(0)=0 .
\end{aligned}
$$

By a suitable normalization, we may also assume

$$
\left|p_{N}\right|^{2}-\mid q_{N} \|^{2} \equiv 1 \quad \text { on } T \text {. }
$$

For details, see [10, pp. 138-139], for example. By (4), equality (1) also can be written as

$$
B_{N}=\left\{\frac{\bar{q}_{N}}{p_{N}}-\frac{w}{p_{N}\left(p_{N}-q_{N} w\right)}: w \in H^{\infty},\|w\|_{\infty}<1\right\} .
$$

We now fix $z \in D$. We define $\bar{q}_{N} / p_{N}$ at $z$ by means of the Poisson integral, and then study the linear transformation

$$
T_{z}: w \rightarrow \frac{\bar{q}_{N}}{p_{N}}(z)-\frac{w}{p_{N}(z)\left(p_{N}(z)-q_{N}(z)\right)},
$$

which necessarily takes $D$ into itself. This is one of the central ideas in Nevanlinna's paper [8].

The radius of the disc $T_{z}(D)$ is easily computed to be

$$
\rho_{N}(z)=\left(\left|p_{N}(z)\right|^{2}-\left|q_{N}(z)\right|^{2}\right)^{-1} \text {. }
$$

Since $B_{F}$ has more than one member, we can find $z \in D, \delta>0$ and an integer $N_{0}$ such that

$$
\rho_{N}(z)>\delta, \quad N \geqslant N_{0} .
$$

By (2)-(4) and Schwartz's lemma, we can thus conclude that the sequence $\left\{\left|p_{N}(z)\right|\right\}_{N}$ is bounded. Applying the Harnack principle to the positive harmonic function $\log \left|p_{N}\right|$ in $D$, we get that $\left\{p_{N}\right\}$ is uniformly bounded on compact subsets of $D$. We can thus find subsequences $\left\{p_{N_{k}}\right\}$ and $\left\{q_{N_{k}}\right\}$ which are uniformly convergent on compact subsets of $D$ to functions $p$ and $q$ respectively. We may also assume that there is a function $r \in L^{\infty}$ such that $\bar{q}_{N_{k}} / p_{N_{k}} \rightarrow r$ in the $w^{*}$-topology.

Since $|q(z)|<|p(z)|, z \in D$, and $p^{-1} \in H^{\infty}$, both $p$ and $q$ have nontangential limits almost everywhere on $T$ by Fatou's theorem [4, p. 13]. 
From the representation formula (5), it follows by an easy normal family argument that

$$
B_{F}=\left\{r-\frac{w}{p(p-q w)}, w \in H^{\infty},\|w\|_{\infty}<1\right\} .
$$

If we knew that $r=\bar{p} / q$ and $|p|^{2}-\left|q^{2}\right|=1$ held almost everywhere on $T$, the formula for $B_{F}$ given by Adamjan, Arov and Krein would easily follow from (6).

To complete our proof, we shall therefore show that $\left\{p_{N_{k}}\right\}$ and $\left\{q_{N_{k}}\right\}$ converge in measure on $T$.

To obtain such a conclusion, we claim

$$
\begin{aligned}
& \text { If } w=w_{\alpha}=e^{i \alpha}, \alpha \text { real, then }\left|r-w_{\alpha} / p\left(p-q w_{\alpha}\right)\right|=1 \\
& \text { almost everywhere on } T \text {. }
\end{aligned}
$$

Assume the claim is proved, and put $I_{\alpha}=r-w_{\alpha} / p\left(p-q w_{\alpha}\right)$. Define also

$$
I_{\alpha, k}=\frac{\bar{q}_{N_{k}}}{p_{N_{k}}}-\frac{w_{\alpha}}{p_{N_{k}}\left(p_{N_{k}}-q_{N_{k}} w_{\alpha}\right)}, \quad k=1,2, \ldots
$$

Following Heins' argument [6, p. 256], we see that $I_{\alpha, k} \rightarrow I_{\alpha}$ in $L^{2}(d \theta)$ as $k \rightarrow \infty$.

If we pick three distinct values of $\alpha$ in $[0,2 \pi)$, we easily deduce that

$$
p_{N_{k}} \rightarrow p \text { and } q_{N_{k}} \rightarrow q,
$$

where the convergence is in measure on $T$.

It remains to prove the claim (*). This claim is of course an immediate consequence of the formula we are trying to establish. Recently J. Garnett [5] has given a proof using methods from functional analysis. A result like (*) was apparently first proved by $R$. Nevanlinna in connection with the Pick-Nevanlinna interpolation problem [8, Satz 7].

Since we want to give a proof along the lines of classical complex analysis, we follow Nevanlinna's idea in [8, pp. 49-51]. So we assume $\left|I_{\alpha}\right|<1-2 r<1$ on a set $E$ of positive linear measure, and shall try to obtain a contradiction.

We put $I_{k}=I_{\alpha} * \sigma_{N_{k}}, k=1,2, \ldots$, and write

$$
I_{k}=\frac{\bar{q}_{N_{k}}-\bar{p}_{N_{k}} w_{k}}{p_{N_{k}}-q_{N_{k}} w_{k}}=\frac{\bar{q}_{N_{k}}}{p_{N_{k}}}-\frac{w_{k}}{p_{N_{k}}\left(p_{N_{k}}-q_{N_{k}} w_{k}\right)}
$$

where $w_{k} \in H^{\infty},\left\|w_{k}\right\|_{\infty} \leqslant 1, k=1,2, \ldots$

Since $I_{k} \rightarrow I$ almost everywhere on $T$, there is a subset $E_{1} \subset E$ of positive measure, such that $\left|I_{k}\right|<1-r$ on $E_{1}$ if $k \geqslant K_{0}$. Following Nevanlinna on pp. 49-50 in [8], we can also deduce that

$$
\left|q_{N_{k}} / p_{N_{k}}\right|<t<1
$$

on a subset $E_{2}$ of $E_{1}$ having positive measure. If $\left[z_{1}, z_{2}\right]$ denotes the noneuclidean distance between $z_{1}$ and $z_{2}$ in $D$, we have from ( 7 ) that

$$
\left[w_{k}\left(e^{i \theta}\right), 0\right]=\left[I_{k}, \frac{\bar{q}_{N_{k}}}{p_{N_{k}}}\right]<\left[I_{k}, 0\right]+\left[\frac{\bar{q}_{N_{k}}}{p_{N_{k}}}, 0\right]<1-r+t
$$

for $k$ sufficiently large and $e^{i \theta} \in E_{2}$. But this is a contradiction. Using (7) and the above expression for $I_{\alpha}$, it is easy to see that $w_{k}(0) \rightarrow w_{\alpha}$ as $k \rightarrow \infty$. Since 
$\left\|w_{k}\right\|_{\infty}<1$, we must have that $w_{k} \rightarrow w_{\alpha}$ uniformly on compact subsets of $D$. Since $w_{\alpha}$ is a constant of modulus one, we can clearly not have $\left|w_{k}\right|<1-t_{0}<1$ on $E_{2}$ if $k$ is sufficiently large; but this is exactly what (8) tells us. The proof is complete.

ADDED IN PROOF (January 22, 1981). The author has recently learned of a proof due to John Garnett of the result of Adamjan, Arov and Krein which is different both from the original proof and from the one above. It can be found in Chapter IV, \$5 of Garnett's forthcoming book, Bounded analytic functions (Academic Press).

\section{REFERENCES}

1. V. M. Adamjan, D. Z. Arov and M. G. Krein, Infinite Hankel matrices and generalized problems of Caratheodory-Fejér and F. Riez, Functional Anal. Appl. 2 (1968), 1-18.

2. __ Infinite Hankel matrices and generalized problems of Caratheodory-Fejer and I. Schur, Functional Anal. Appl. 2 (1968), 269-281.

3. Analytic properties of Hilbert-Schmidt pairs for a Hankel operator and the generalized Schur-Takagi problem, Mat. USSR Sb. 15 (1971), 31-73.

4. P. L. Duren, Theory of $H^{P}$-spaces, Academic Press, New York, 1970.

5. J. Garnett, Two remarks on interpolation by bounded analytic functions, Proc. of Pelczynski Conference at Kent State University, Lecture Notes in Math., vol. 604, Springer-Verlag, Berlin and New York, 1977.

6. M. Heins, On W.S.N. functions and a theorem of Nevanlinna concerning bounded analytic functions, Kodai Math. Sem. Reports, 26 (1975), 245-257.

7. R. Nevanlinna, Über beschränkte Funktionen die in gegebenen Punkten vorgeschribene Werte annehmen, Ann. Acad. Sci. Fenn. Ser. B 15 (1919), 1-71.

8. __ Über beschränte analytische Funktionen, Ann. Acad. Sci. Fenn. Ser. A 32 (1929), 1-75.

9. I. Schur, Über Potenzreihen, die im Inneren des Einheitskreise beschränkt sind I, J. Math. 147 (1917), 205-232.

10. , Uber Potenzreihen, die im Inneren des Einheitskreise beschräkt sind I, J. Math. 148 (1918), $122-145$

Agder Distriktshogskole, Postboks 607, N-4601 Kristiansand, Norway 